\title{
The Globular Cluster Terzan 7 in the Sagittarius Dwarf Spheroidal Galaxy
}

\author{
Enrico V. Held \\ Osservatorio Astronomico di Padova, I-35122 Padova, Italy \\ Alfred Rosenberg \\ Instituto de Astrofísica de Canarias, 38200 La Laguna, Spain
}

Ivo Saviane

European Southern Observatory, Casilla 19001, Santiago 19, Chile

Yazan Momany

Dipartimento di Astronomia, Univ. di Padova, I-35122 Padova, Italy

\begin{abstract}
We present deep $V, I$ photometry of the globular cluster Terzan 7, a probable member of the globular cluster system of the Sagittarius dwarf spheroidal galaxy. The metallicity, estimated from a new method based on analytic RGB fits, agrees with previous estimates based on color-magnitude diagrams $([\mathrm{Fe} / \mathrm{H}]=-0.9 \pm 0.1 \mathrm{dex})$. This result confirms a discrepancy between photometric and spectroscopic determinations. Using both the horizontal and vertical methods to estimate relative ages, we confirm that the age of Terzan 7 is about $70 \%$ that of 47 Tuc. A rich population of blue stragglers is found, strongly concentrated toward the center of the cluster.
\end{abstract}

\section{Introduction}

The Sagittarius galaxy (Sgr) is the nearest dwarf spheroidal, and shows clear signs of interaction with the Milky Way (Ibata et al. 1994). Terzan 7 appears to be the youngest and most metal-rich of the four clusters associated with Sgr (Buonanno et al. 1995). Measuring its age and metal content is clearly important to trace the evolution of its parent galaxy and the Milky Way halo. Previous photometric estimates of its metal abundance are in the range $[\mathrm{Fe} / \mathrm{H}]=-1$ (Buonanno et al. 1995) to $[\mathrm{Fe} / \mathrm{H}]=-0.8$ (Sarajedini \& Layden 1997), while measurements of the CaII triplet line strengths yield $[\mathrm{Fe} / \mathrm{H}] \simeq-0.4$ (Da Costa \& Armandroff 1995) and high-resolution measurements give $[\mathrm{Fe} / \mathrm{H}] \simeq-0.6$ (Wallerstein et al. 2002, this conference). Such a disagreement between the photometric and spectroscopic estimates is common to several halo clusters. As a contribution to pinpoint the properties of the Sgr globular cluster system, we have obtained deep VI photometry of Terzan 7 at the ESO NTT. Preliminary results on the 


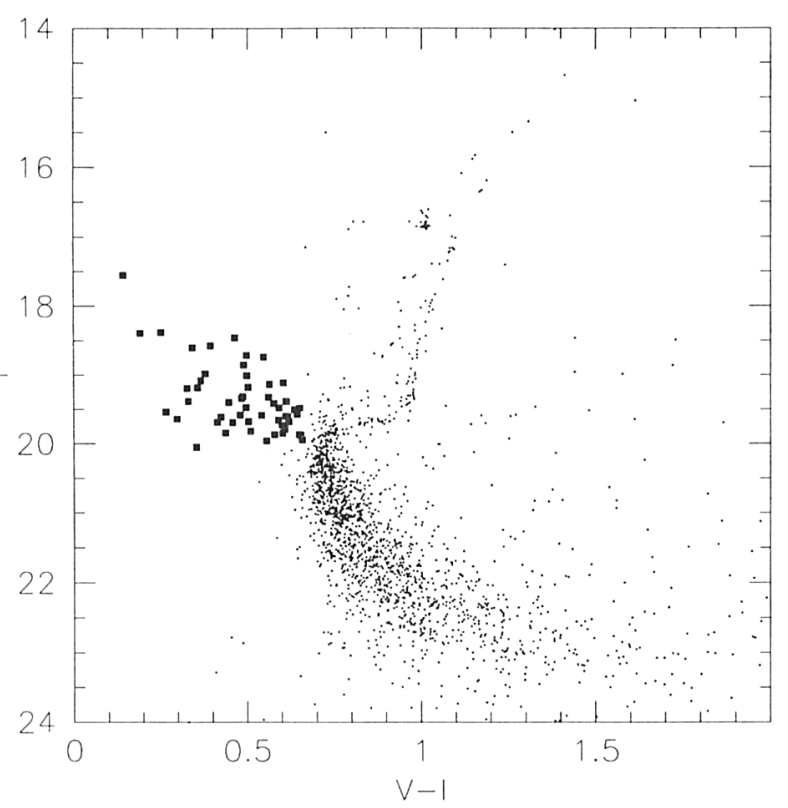

Figure 1. The $I,(V-I)$ color-magnitude diagram of Terzan 7. Dots represent stars within $1^{\prime}$ from the cluster center, filled squares are the blue stragglers found within the same radius.

age, metallicity and stellar content of Terzan 7 are reported here based on these new observations.

\section{Results}

Observations of the globular cluster Terzan 7 were obtained on the night of 30 Sept. 1999 using EMMI (f.o.v. 9'.2×8'8) at the ESO NTT telescope. The seeing was good $\left(\sim 0.8^{\prime \prime}\right)$ and the sky was clear. The data set consists of two 5 min exposures and one $1 \mathrm{~min}$ exposure in each band. The two images in each filter were pre-processed and co-added in a standard way using IRAF, and stellar photometry was obtained using the PSF-fitting program ALLSTAR (Stetson \& Harris 1988). The data were calibrated using observations of Landolt's (1992) standard fields. A comparison of our calibrated CMD with the results of Sarajedini \& Layden (1997) indicates good agreement of the zero points.

Figure 1 shows the $I,(V-I)$ color-magnitude diagram (CMD) of stars in the field of Terzan 7 . To reduce the foreground contamination by bulge/disk stars, only stars within a $1^{\prime}$ radius from the center have been plotted. A relatively low reddening, $\mathrm{E}(B-V)=0.06 \pm 0.02$, was adopted on the basis of a color comparison of the red horizontal branch (HB) in Terzan 7 and the young halo cluster Pal 12 (Rosenberg et al. 1998). A preliminary metallicity estimate, $[\mathrm{Fe} / \mathrm{H}]=-0.9 \pm 0.1$ dex on the Zinn \& West's (1984) scale was obtained by interpolating across a grid of analytic fitting functions modeling the RGB's of Galactic globular clusters (see 
Saviane et al. 2000). A similar metallicity was estimated from the slope of the RGB using the Hartwick (1968) S-index with the re-calibration of Saviane et al. (2000). This result confirms the disagreement with the metallicities measured by both intermediate-resolution spectroscopy in the CaII triplet region (Da Costa \& Armandroff 1995) and high-resolution abundance analysis (Wallerstein et al. 2001 , this conference), which are more metal-rich by $\gtrsim 0.3$ dex. The sparsely populated RGB of Terzan 7, along with the high foreground contamination, represent the main problems for any photometric $[\mathrm{Fe} / \mathrm{H}]$ measurements, and a membership confirmation based on spectroscopy and/or proper motions would be important in this respect.

The magnitude and color of the main-sequence turnoff confirm that Terzan 7 is definitely younger than old Galactic globular clusters (see Buonanno et al. 1995; Sarajedini \& Layden 1997). We used both the horizontal and vertical methods to estimate relative ages. A comparison of the CMD's of Terzan 7 and Pal 12 suggests that these clusters are nearly coeval, with age about $70 \%$ that of 47 Tuc. The ages of Terzan 7 (this work) and Pal 12 (Rosenberg et al. 1998) were compared with those of clusters in the large sample of Rosenberg et al. (2000), as a function of both metallicity and Galactocentric distance. The properties of Terzan 7 and Pal 12 appear to be very similar. Interestingly, this agrees with the suggestion of Dinescu et al. (2000) that, based on its orbital characteristics, Pal 12 might have been captured from Sagittarius about $1.7 \mathrm{Gyr}$ ago.

Terzan 7 is known to contain a rich population of blue straggler stars (BSS) concentrated toward the center of the cluster (Fig. 1; see also Buonanno et al. 1995). Our new data allow an analysis of the spatial distribution and luminosity range of BSS out to $\sim 6^{\prime}$ from the cluster center. The high central concentration of the blue stragglers is confirmed by a K-S test indicating that the surface density profiles of BSS and subgiant/giant stars are different at a very high (> $99 \%)$ confidence level.

\section{References}

Buonanno, R., Corsi, C. E., Pulone, L., Fusi-Pecci, F., Richer, H. B., \& Fahlman, G. C. 1995, AJ, 109, 663

Da Costa, G. S., \& Armandroff, T. E. 1995, AJ, 109, 2533

Dinescu, D. I., Majewski, S. R., Girard, T. M., \& Cudworth, K. M. 2000, AJ, 120,1892

Hartwick, F. D. A. 1968, ApJ, 154, 475

Ibata, R. A., Gilmore, G., Irwin, M. J. 1994, Nature, 370, 194

Landolt, A. U. 1992, AJ, 104, 340

Rosenberg, A., Saviane, I., Piotto, G., \& Held, E. V. 1998, A\&A, 339, 61

Rosenberg, A., Piotto, G., Saviane, I., \& Aparicio, A. 2000, A\&AS, 144, 5

Sarajedini, A., \& Layden, A. 1997, AJ, 113, 264

Saviane, I., Rosenberg, A., Piotto, G., \& Aparicio, A. 2000, A\&A, 355, 966

Stetson, P. B., \& Harris, W. E. 1988, AJ, 96, 909

Zinn, R., \& West, M. J. 1984, ApJS, 55, 45 